\title{
Urinary excretion reflects lung deposition of aminoglycoside aerosols in cystic fibrosis
}

\author{
P-F. Dequin*, F. Faurisson", E. Lemarié*, F. Delatour", S. Marchand", C. Valat*, E. Boissinot*, \\ C. de Gialluly ${ }^{+}$, P. Diot*
}

Urinary excretion reflects lung deposition of aminoglycoside aerosols in cystic fibrosis. P-F. Dequin, F. Faurisson, E. Lemarié, F. Delatour, S. Marchand, C. Valat, E. Boissinot, C.de Gialluly, P. Diot. (C)ERS Journals Ltd 2001.

ABSTRACT: Using nebulization to deliver aminoglycosides may be of benefit in cystic fibrosis (CF) patients colonized by Pseudomonas aeruginosa. However, one problem with this route is the absence of clinical parameters allowing estimation of the mass of drug deposited in the lungs (MDL). The aim of this study was to assess whether aminoglycoside excretion in the urine reflects the MDL.

Fourteen studies were performed in seven CF patients. Amikacin was mixed with albumin labelled with ${ }^{99 m}$ Tc and nebulized with an ultrasonic nebulizer. The MDL was determined by the mass-balance technique. Urine was collected during the $24 \mathrm{~h}$ following inhalation and was assayed for amikacin by fluorescence polorization immunoassay (FPIA).

The mean \pm SEM MDL was $14.0 \pm 2.2 \%$ of the nebulizer charge. The mean \pm SEM amount of amikacin excreted in the urine was $20.9 \pm 4.5 \mathrm{mg}$ and correlated with the MDL ( $r=0.93 ; p=0.0001)$. There was, however, wide intersubject variability in both deposition and excretion in the urine.

Monitoring excretion of aminoglycosides in the urine allows noninvasive estimation of the mass of drug deposited in the lung in cystic fibrosis patients, which might be useful to assess the dose-response relationship in groups of patients, but intersubject variability prevents its use for individual follow-up.

Eur Respir J 2001; 18: 316-322.

\begin{abstract}
*Inserm EMI-U 00-10, University of Tours, Medical Intensive Care Unit and Dept of Respiratory Diseases, Bretonneau University Hospital, Tours,

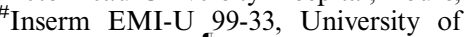
Paris 7, Paris, "Dept of Childhood Diseases and Cystic Fibrosis Unit, Clocheville University Hospital, Tours, and ${ }^{+}$Microbiology Laboratory, Trousseau University Hospital, Tours, France.
\end{abstract}

Correspondence: P. Diot, Service de Pneumologie, CHU Bretonneau, 37044 Tours cedex 1, France.

Fax: 33247473882

Keywords: Aerosol aminoglycoside cystic fibrosis pharmacokinetics urine

Received: September 301999 Accepted after revision April 112001

This study was supported by Association Française de Lutte contre la Mucoviscidose.
Aminoglycosides are among the most effective antibiotics used to treat Pseudomonas aeruginosa colonization in cystic fibrosis $(\mathrm{CF})$ patients $[1,2]$. RAMSEY et al. [3] have demonstrated that 24-week treatment with aerosolized tobramycin improves pulmonary function in clinically stable $\mathrm{CF}$ patients colonized by $P$. aeruginosa, decreases the density of bacteria in the sputum, and decreases the risk of hospitalization [3]. The efficacy of aminoglycoside aerosols in treating $P$. aeruginosa colonization in $\mathrm{CF}$ patients is intuitively considered to depend upon the mass of antibiotic deposited in the lungs, but this has not been demonstrated, and it is difficult to measure in clinical practice. Indeed, determination of the amount of drug deposited in the lungs requires radiolabelling of the drug and use of a gamma-camera, and this is not routinely possible. Aminoglycosides are resorbed into the plasma after deposition in the lungs and then excreted in the urine, but little is known about the pharmakokinetics. Blood concentrations are low [4] and they do not appear to be promising markers of absorption, and hence, deposition of aminoglycosides. An alternative approach might be monitoring aminoglycoside excretion by collecting and pooling urine over a period of time after aminoglycoside inhalation.

The aim of the present study was to test the hypothesis that aminoglycoside excretion in the urine reflects the deposition of the drug in the lungs, which would make the urinary pharmacokinetics of the drug a potential tool to assess a dose-response relationship.

\section{Patients and methods}

\section{Patients}

Seven CF patients were enrolled after obtaining signed informed consent from themselves (adult patients) or from their parents, associated for children with their own oral consent. The study was approved by the Ethics Committee of Tours University. Patients had to fulfil all of the following inclusion criteria: age $>6$ yrs; colonization by amikacin-sensitive $P$. aeruginosa (defined by the presence of $1 \times 10^{3}$ or more colony forming units $\cdot \mathrm{mL}^{-1}$ on sputum obtained 
from a deep cough on three occasions over $>1 \mathrm{yr}$ ); $\leqslant 10 \%$ change in forced expiratory volume in one second (FEV1) on the day of the deposition study (compared to the reference value measured one month before); and absence of any infectious exacerbation. Patients were not included if they used any other antibiotic aerosol as a part of regular treatment. The main characteristics of the population studied are presented on table 1 . A total of 14 deposition studies was performed for the seven patients (two studies for all child patients except one, and three studies for the adult patient). There was a 3-month interval between studies for each individual. The studies were performed one month after completing a regular course of intravenous antibiotics.

\section{Pulmonary function tests}

FEV1, forced vital capacity (FVC) and forced midexpiratory flow (FEF25-75) were measured before and after amikacin inhalation using a constant-pressure plethysmograph (Sensor Medics 2,800, USA). These parameters were expressed as \% predicted [6].

\section{Lung volume assessment}

Ventilation studies with ${ }^{133} \mathrm{Xe}$ were performed during tidal breathing. The patient was seated in front of the gamma-camera (Pho Gamma Searle, USA), set for ${ }^{133} \mathrm{Xe}$, and breathed into a bag-box spirometer (Xenamatic, Diversified Diagnostic Products, Inc., Houston, TX, USA), whilst wearing a noseclip. A $10 \mathrm{mCi}^{133} \mathrm{Xe}$ dose (XE-133-G-2B, Cis Bio International, France) was injected into the bag-box, whilst the patient breathed normally in order to reach equilibrium, which was demonstrated by continuous dynamic counting over periods of $15 \mathrm{~s}$, and 20 images of $3 \mathrm{~s}$ were then acquired on a $64 \times 64$ matrix. Lung outlines were drawn over the ${ }^{133} \mathrm{Xe}$ equilibrium scan, i.e. the sum of the 20 images acquired at the equilibrium state (computer Simis, Siemens, Germany). These outlines were then superimposed on the ${ }^{99 \mathrm{~m}} \mathrm{Tc}$ deposition image.

\section{Amikacin aerosol}

Amikacin sulphate (Bristol-Myers Squibb, France) was dissolved in sterile water to a concentration of $125 \mathrm{mg} \cdot \mathrm{mL}^{-1}$, and mixed with $0.3 \mathrm{~mL}$ of a solution of $10 \mathrm{mg}$ human serum albumin (TCK2, Cis Bio-International, France) labelled with ${ }^{99 \mathrm{~m}}$ Tc. The aerosols were nebulized with a SAM LS ${ }^{\mathrm{TM}}$ ultrasonic nebulizer (Syst'Am, France), operating at a frequency of $2.4 \mathrm{MHz}$ and used at its maximum intensity. It has been demonstrated in a previous study, that there is no leak during expiration with this device, that labelling the drug does not alter the aerosol aerodynamic properties, and that ${ }^{99 \mathrm{~m}} \mathrm{Tc}$ counting accurately reflects amikacin mass in the aerosol [7]. The mass median aerodynamic diameter (MMAD) of the aerosol was $3.0 \mu \mathrm{m}$, and geometric standard deviation (GSD) was 1.7.

\section{Amikacin aerosol deposition study}

A solution of $1.0 \mathrm{~g}$ amikacin in $8 \mathrm{~mL}$ sterile water was used for children, and a solution of $1.5 \mathrm{~g}$ amikacin in $12 \mathrm{~mL}$ sterile water for the adult patient. The solutions were radiolabelled as described above. The activity placed in the nebulizer was counted using a gamma-well counter (Capintec, CRC Aries, France). A 300-s image was acquired to take into account the room background in the deposition analysis. The patient then inhaled the aerosol in a sitting position,

Table 1.- Pulmonary function and breathing patterns

\begin{tabular}{|c|c|c|c|c|c|c|c|c|}
\hline Patient number & Age yrs & $\begin{array}{c}\text { Study } \\
\text { number }\end{array}$ & $\begin{array}{c}\text { SHWACHMAN's } \\
\text { score [5] }\end{array}$ & FEV1 \% & FVC \% & FEF25-75 \% & $V \mathrm{~T} \mathrm{~mL}$ & $\mathrm{RR} \min ^{-1}$ \\
\hline \multirow[t]{2}{*}{1} & \multirow[t]{2}{*}{11} & 1a & 83 & 87.3 & 100.1 & 75.5 & 790 & 14 \\
\hline & & $1 \mathrm{~b}$ & 83 & 69.5 & 101.6 & 40.7 & 550 & 16 \\
\hline \multirow[t]{2}{*}{2} & \multirow[t]{2}{*}{9} & $2 \mathrm{a}$ & 68 & 50.3 & 85.6 & 19.2 & ND & ND \\
\hline & & $2 b$ & 83 & 68.7 & 99.1 & 30.3 & 440 & 21 \\
\hline \multirow[t]{3}{*}{3} & \multirow[t]{3}{*}{31} & $3 a$ & ND & 28.9 & 60.5 & 10.5 & 980 & 11 \\
\hline & & $3 b$ & ND & 32.0 & 68.6 & 11.1 & 1260 & 11 \\
\hline & & $3 \mathrm{c}$ & ND & 28.1 & 68.0 & 9.7 & ND & ND \\
\hline \multirow[t]{2}{*}{4} & \multirow[t]{2}{*}{10} & $4 a$ & 82 & 82.5 & 100.6 & 57.9 & 730 & 10 \\
\hline & & $4 \mathrm{~b}$ & 82 & 65.7 & 88.1 & 36.1 & ND & ND \\
\hline \multirow{2}{*}{5} & \multirow[t]{2}{*}{8} & $5 a$ & 89 & 82.5 & 96.2 & 67.5 & 600 & 11 \\
\hline & & $5 b$ & 83 & 83.6 & 104.9 & 55.7 & 360 & 12 \\
\hline 6 & 8 & $6 a$ & 97 & 99.9 & 106.5 & 88.8 & 380 & 13 \\
\hline \multirow[t]{2}{*}{7} & \multirow[t]{2}{*}{17} & $7 \mathrm{a}$ & 58 & 24.6 & 34.4 & 9.3 & ND & 15 \\
\hline & & $7 b$ & 55 & 21.5 & 26.0 & 13.2 & 370 & 12 \\
\hline Median & 10 & & 83 & 68.7 & 96.2 & 36.1 & 600 & 12.5 \\
\hline Mean & 13.4 & & 78.5 & 58.9 & 81.4 & 37.5 & 690 & 13.5 \\
\hline $95 \% \mathrm{CI}$ of the mean & & & $69.9-87.1$ & $43.2-74.7$ & $66.3-96.6$ & $21.8-53.3$ & $480-910$ & $11.5-15.5$ \\
\hline
\end{tabular}

FEV1: forced expiratory volume in one second; FVC: forced vital capacity; FEF25-75\%: forced mid-expiratory flow; VT: tidal volume; RR: respiratory rate; ND: not determined; CI: confidence interval; FEV1, FVC and FEF25-75 are expressed as the percentage of the predicted values. 
whilst wearing a noseclip. The tidal volume and respiratory frequency were continuously monitored during the 10-min nebulization, using a pneumotachograph (Fleisch no. 3, Fleisch, Lausanne, Switzerland) placed on the expiratory line of the nebulizer and connected to a polygraph recorder. This system was calibrated for each study. A low resistance filter (German Sciences, Ann Arbor, MI, USA) was placed on the expiratory line in order to capture all the aerosol particles exhaled by the patient. A 60 -s static image was acquired immediately after the inhalation after drinking water to wash activity out of the pharynx into the stomach.

\section{Quantification of aerosol deposition}

The activity deposited on the expiratory filter and the activity remaining in the nebulizer reservoir and lines at the end of nebulization were counted in the gamma-well counter. Counts were then corrected for ${ }^{99 \mathrm{~m}}$ Tc physical decay. The inhaled mass was calculated based on the difference between the activity placed in the reservoir of the nebulizer before nebulization, and the activity remaining in the nebulizer at the end of nebulization, taking into account the relationship between amikacin mass and ${ }^{99 \mathrm{~m}} \mathrm{Tc}$ activity in the aerosol. The exhaled activity was equal to the activity deposited on the expiratory filter. The inhaled fraction (IF) was equal to the ratio of the inhaled activity to the activity placed in the nebulizer before nebulization. The inhaled mass of amikacin was the product of IF multiplied by the mass of drug deposited in the nebulizer. The deposited fraction (DF) was defined as the fraction of the radiolabelled aerosol deposited in the patient [8], and was provided by the following equation:

$$
\begin{aligned}
\mathrm{DF}= & (\text { radioactivity inhaled }- \text { radioactivity } \\
& \text { exhaled }) / \text { radioactivity inhaled }
\end{aligned}
$$

The deposited mass of amikacin was the product of the inhaled mass multiplied by the DF [9] and was distributed between the lungs and the stomach. The fraction deposited in the lungs was determined on the ${ }^{99 \mathrm{~m}}$ Tc deposition image. This fraction, multiplied by the deposited mass of amikacin, defined the deposited mass of drug in the lungs (MDL), expressed as a percentage of the mass of amikacin placed in the nebulizer (MDL\%), or as the mass of amikacin (MDLmg).

\section{Monitoring of urine pharmacokinetics}

Urine was collected immediately before the amikacin aerosol deposition study and then at each micturition during the $24 \mathrm{~h}$ following inhalation. The patient weighed the urine using an electronic balance (Terraillon, France), kept a sample and recorded the exact time of micturition. Urine samples were stored at $4{ }^{\circ} \mathrm{C}$. At the end of the $24 \mathrm{~h}$ period, all samples were assayed for amikacin and creatinine. Amikacin and creatinine levels were measured by fluorescence polarization immuno-assay (FPIA) (TDx Abbott, Abbott
Park, IL, USA). The total amount of creatinine excreted in the urine allowed determination of whether the urine collected was truly representative of $24 \mathrm{~h}$ urine collection. The elimination flow rate of amikacin excreted in the urine for a definite micturition (Qamik) was calculated as the ratio of the mass of amikacin assayed in the urine, divided by the time elapsed since the previous micturition. The total amount of amikacin excreted in the urine after aerosol delivery (excreted amount, Uamik,total) was determined by assays of each micturition for $24 \mathrm{~h}$ (measured amount, Uamik,24h), then by extrapolation to infinity (extrapolated

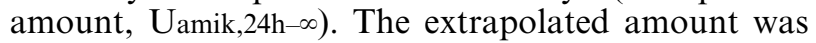
calculated by integration of logarithmic regression of elimination flow versus time, from $24 \mathrm{~h}$ to infinity.

\section{Statistical tests}

The results are presented as mean \pm SEM or, when appropriate, as medians. Pulmonary function tests before and after nebulization were compared using a Wilcoxon test. Correlations were assessed by linear regression. A p-value $<0.05$ was considered significant. As the design of the study involved repeated measurements for the same subjects, correlations were assessed for all measurements (i.e. 14 measurements) and also for the first measurement in each subject (i.e. seven measurements). A Bland and Altman analysis [10] was used to measure agreement between measurement of MDL and its estimation obtained from the urinary excretion of amikacin.

\section{Results}

\section{Nebulization tolerance}

Tolerance of inhalation assessed by clinical judgement was good for all patients, except for patient 2 who developed a cough soon after the start of nebulization. The nebulizer was then set at medium intensity, and there was then good tolerance.

Pulmonary function tests did not change after nebulization (table 2).

\section{Total aerosol deposition}

The IF, DF and MDL per patient are shown in table 3. Mean IF was $19.9 \pm 2.5 \%$, with a wide range

Table 2. - Comparison of pulmonary function tests before and after nebulization of the radiolablled amikacin aerosol using the Wilcoxon test

\begin{tabular}{lccc}
\hline & $\begin{array}{c}\text { Before } \\
\text { nebulization }\end{array}$ & $\begin{array}{c}\text { After } \\
\text { nebulization }\end{array}$ & p-value \\
\hline FEV1 \% & $58.9(43.2-74.7)$ & $57.9(43.2-72.6)$ & 0.40 \\
FVC \% & $81.4(66.3-96.6)$ & $81.2(67.2-95.1)$ & 0.93 \\
FEF25-75\% & $37.5(21.8-53.3)$ & $34.2(22.2-46.1)$ & 0.25 \\
\hline
\end{tabular}

The data are presented as mean $(95 \%$ confidence intervals of the mean). FEV1: forced expiratory volume in one second; FVC: forced vital capacity; FEF25-75 \%: forced midexpiratory flow. 
Table 3. - Determinants of deposition, individual values of lung deposition and urine excretion of amikacin

\begin{tabular}{lccccccr}
\hline Study number & IF $\%$ & DF $\%$ & Lung $\%$ & MDL\% & MDLmg & Uamik,total, $\%$ & Uamik,total,mg \\
\hline la & 14.9 & 99.8 & 32 & 4.7 & 47 & 0.21 & 2.1 \\
1b & 11.7 & 99.0 & 37 & 4.3 & 43 & 0.16 & 1.6 \\
2a & 19.0 & 98.0 & 97 & 18.0 & 180 & 1.84 & 18.4 \\
2b & 12.1 & 93.8 & 80 & 9.0 & 90 & 0.94 & 9.4 \\
3a & 19.0 & 97.0 & 89 & 16.4 & 246 & 2.25 & 33.7 \\
3b & ND & ND & 100 & ND & ND & 0.95 & 14.2 \\
3c & 2.0 & 71.0 & 76 & 1.3 & 20 & ND & ND \\
4a & 33.0 & 98.0 & 77 & 25.2 & 252 & 3.30 & 33 \\
4b & 27.0 & 96.0 & 73 & 18.9 & 189 & 2.34 & 23.4 \\
5a & 20.1 & 98.7 & 67 & 13.3 & 133 & 0.80 & 8 \\
5b & ND & ND & 84 & ND & ND & 5.69 & 56.9 \\
6a & 26.8 & 94.5 & 71 & 19.2 & 192 & 2.54 & 25.4 \\
7a & 28.6 & 90.9 & 76 & 18.5 & 185 & 3.52 & 35.2 \\
7b & 24.0 & 94.0 & 81 & 19.0 & 190 & ND & ND \\
Median & 19 & 97 & 76 & 16.4 & 180 & 2.05 & 20.9 \\
Mean & 19.9 & 94.2 & 74.3 & 14.0 & 147 & 2.05 & 21.8 \\
95\% CI of the mean & $14.3-25.4$ & $89.7-98.7$ & $63.2-85.4$ & $9.3-18.7$ & $97-198$ & $0.84-2.93$ & $11.5-32.1$ \\
\hline
\end{tabular}

IF: inhaled fraction; DF: deposited fraction; Lung: pulmonary deposition expressed as a percentage of the deposited mass; MDL: mass of drug deposited in the lungs, expressed as a percentage of the mass of amikacin initially placed in the nebulizer (MDL\%) or as a mass (MDLmg); Uamik,total: total amount of amikacin excreted by the urine, expressed as a percentage of the mass of amikacin initially placed in the nebulizer (Uamik,total,\%) or as a mass (Uamik,total,mg); ND: not determined; CI: confidence interval.

from 2.0-33.0\%. Mean DF was $94.2 \pm 2.1 \%$ (from $71.0 \%-99.8 \%$ ). Mean MDL $\%$ was $14.0 \pm 2.2 \%$ (from $1.3 \%-25.2 \%)$.

MDL $\%$ correlated with IF: MDL $\%=0.79 \mathrm{IF}=-1.73$, $r=0.93, p=0.0001$ (fig. 1). This finding was insensitive to restriction to the first measurement for each patient $(\mathrm{r}=0.85, \mathrm{p}=0.016)$. IF, DF and $\mathrm{MDL} \%$ were not correlated with age, Shwachman's score, breathing pattern or lung function tests.

Relationship between urinary amikacin excretion and aerosol deposition

The mean total amount of amikacin excreted in the urine (Uamik,total,mg) was $21.8 \pm 4.7 \mathrm{mg}$ (1.6 to $56.9 \mathrm{mg}$ ). Uamik,total,\% represented $1.89 \pm 0.5 \%(0.16-5.69 \%)$ of

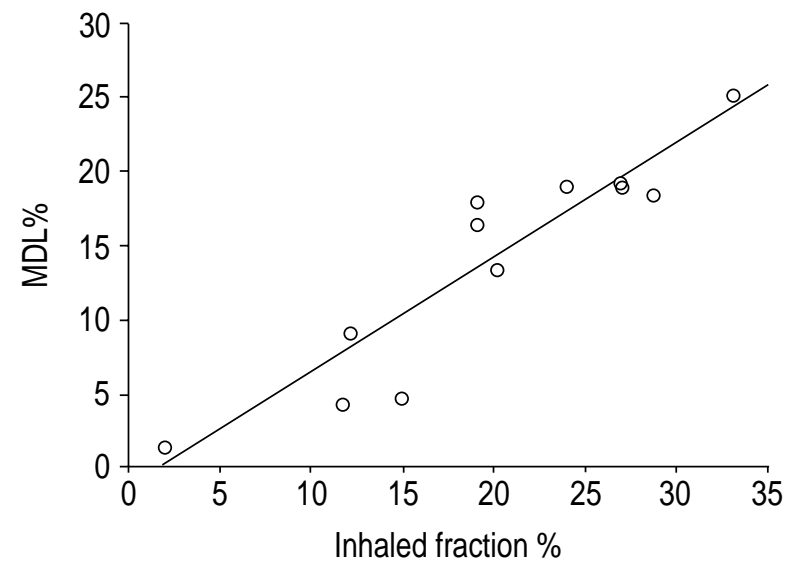

Fig. 1.-Relationship between inhaled fraction and the mass of drug deposited in the lungs (MDL\%). Both parameters are expressed as the percentage of the amount of amikacin placed in the nebulizer prior to nebulization. Regression line is shown $(\mathrm{y}=0.79 \mathrm{x}-1.73 ; \mathrm{r}=0.93 ; \mathrm{p}=0.0001)$. the mass of amikacin placed in the nebulizer prior to nebulization. This percentage correlated with IF $(\mathrm{r}=0.78, \mathrm{p}=0.008)$ and MDL\% $(\mathrm{r}=0.77, \mathrm{p}=0.009)$.

Uamik,total,mg was strongly correlated with MDLmg (fig. 2): Uamik, total,mg=(0.16 MDLmg)-6.21 ( $\mathrm{r}=0.93$, $\mathrm{p}=0.0001$ ). This finding was insensitive to restriction to the first measurement for each patient ( $\mathrm{r}=0.9$, $\mathrm{p}=0.006$ ). It was also insensitive to restriction to the fraction of excreted amikacin actually assayed, i.e. Uamik,24h,mg ( $r=0.92, p=0.0002)$. Uamik,24h,mg represented $94.8 \%$ of Uamik,total,mg.

The correlation equation determined by linear regression between MDLmg and Uamik,24h,mg was used to calculate MDLmg (MDLmg,calc) from Uamik,24h,mg: MDLmg,calc $=(5.48$ Uamik,24h,mg $)+57.7$. As described by Bland \& Altman [10], a graph was drawn (fig. 3) presenting the average MDL versus differences

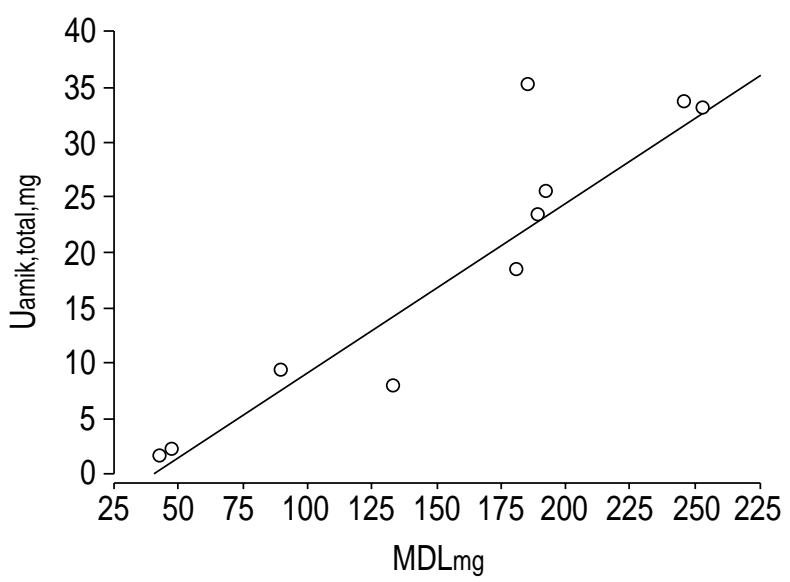

Fig. 2.-Relationship between the mass of drug deposited in the lungs expressed as mass of amikacin in mg (MDLmg) and the total amount of amikacin excreted in the urine in $\mathrm{mg}$ (Uamik,total,mg). Regression line is shown ( $y=0.16 x-6.21 ; r=0.93$; $p=0.0001)$. 


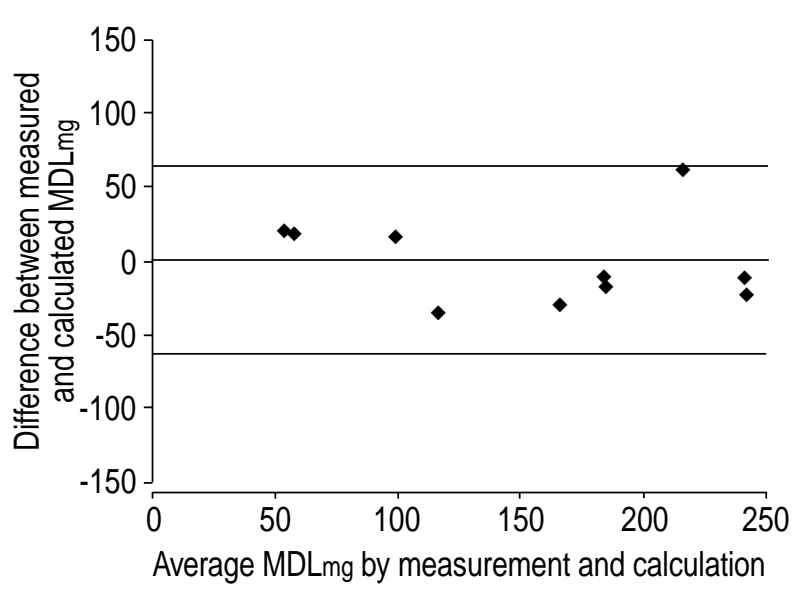

Fig. 3.-Relationship between the average mass of drug deposited in the lungs (MDL) and differences between mass of drugs deposited in the lungs in $\mathrm{mg}$ (MDLmg) and calculated mass of drugs deposited in the lungs in $\mathrm{mg}$ (MDLmg,calc) (Y-axis), according to BLAND and ALTMAN [10]. Horizontal lines represent the mean \pm 2 SD difference.

between MDLmg and MDLmg,calc. All differences between assessments of MDL lay within \pm 2 SD of the mean.

\section{Discussion}

The present study demonstrated that MDL was correlated with IF. More interestingly, amikacin excreted in the urine was strongly correlated with IF and MDL, demonstrating that amikacin excretion in the urine reflects the amount of drug deposited in the lungs.

Amikacin is a semisynthetic aminoglycoside which is effective against aerobic Gram negative bacteria isolated from $\mathrm{CF}$ patients, including $P$. aeruginosa [11]. Most studies involving aerosolized aminoglycosides have been performed with gentamicin [4] or tobramycin $[3,12]$. Some in vitro studies demonstrated that isolates of $P$. aeruginosa are more often resistant to amikacin than to tobramycin [13]. However, in view of the high doses that were used, it was assumed that the concentrations of antibiotic obtained in the sputum were very high, as demonstrated by ILOwITE [4] with gentamicin. The notion of resistance as determined in vitro was therefore of little value, local concentrations being much higher than the minimal inhibitory concentrations. Furthermore, a sulphitefree form of tobramycin was not commercially available in France. All other aminoglycosides, but not the commercially available form of amikacin in France (Amiklin $\mathbb{R})$, contain sulphites that may be responsible for severe allergic side effects [14]. This is particularly important in $\mathrm{CF}$ patients who are known to have frequent bronchial hyperreactivity [15] and are at risk of bronchial allergy [16], and this is why the present study was conducted with amikacin. In the present study, tolerance of inhalation was good for all patients and pulmonary function tests were unchanged after nebulization.

The efficacy of therapeutic aerosols might be related to the amount of drug deposited in the lungs. Unfortunately, little information is available concerning aerosol deposition in the lungs of CF patients. The IF is a characteristic of the nebulizer, while the DF depends on the anatomy of the airways and breathing patterns [9]. In the present study, the DF was higher than $90.0 \%$ for all patients studied, except one. Such a high DF has been reported in clinical studies for chronic obstructive pulmonary disease (COPD) patients [17], in an animal model of chronic bronchitis [18] and in an in vitro airway model with excessive mucus secretion [19]. This suggests that significant aerosol deposition is obtained because airway obstruction in COPD and CF patients enhances deposition of aerosol particles. In patients of the present study, mean MDL \% was $14.0 \%$ of the mass of amikacin placed in the nebulizer $(95 \%$ confidence interval $9.3-18.7 \%$ ), corresponding to $20-252 \mathrm{mg}$ of amikacin deposited in the lungs. However, the MDLmg was $\geqslant 100 \mathrm{mg}$ in all studies, except three. Of these three studies, two were performed in the same patient (patient 1), who had high gastric activity after pharyngeal washing, suggesting high upper airway deposition. Mean MDL\% was higher than the average amount of gentamicin deposited in the lungs $(7.69 \%)$ in the study of ILOwITE [4]. The presented study used a nebulizer requiring inspiratory flow applied to the inspiratory line, to make the aerosol available for inhalation, probably accounting for higher efficiency by avoiding leakage.

A correlation was found between $\mathrm{MDL} \%$ and IF, but not between MDL $\%$ and other parameters. IF was the most significant parameter of amikacin aerosol deposition in the $\mathrm{CF}$ patients. The correlation between MDL $\%$ and IF (MDL $\%=0.79 I F-1.73$ ) demonstrates that the amount deposited was about $80 \%$ of the inhaled dose, with low interpatient variability of deposition rate (i.e. exhaled fraction). Surprisingly no correlation between breathing pattern and IF or MDL was found, while ILowite [4] found a bell-shaped distribution between deposited dose of gentamicin and minute ventilation. The low amount of antibiotic delivered to the lungs at low minute ventilation by leakage during expiration was explained. Patients in the present study had been especially trained for nebulization, which might have reduced the interpatient variations in breathing pattern. A recent study reporting factors influencing deposition of recombinant human deoxyribonuclease (rhDNase) I in $\mathrm{CF}$, reported a negative correlation between $\mathrm{FEV} 1 \%$ and lung deposition of rhDNase I [20]. In the present study, no correlation between pulmonary function tests and lung deposition of amikacin was found.

The selective inclusion and noninclusion criteria and the experimental design of the present study resulted in the enrolment of a relatively small number of patients. It was therefore decided to perform two measurements in each subject. Although the patients were studied during a stable phase of the disease, changes were observed in respiratory function and aerosol deposition between the measurements, which were performed after an interval of 3 months. However, no correlation was found between changes in respiratory function (e.g. $\Delta \mathrm{FEV} 1 \%$ ) and any 
aerosol deposition parameter (i.e. $\Delta \mathrm{IF}, \Delta \mathrm{MDL} \%$ or $\Delta$ Uamik,total). For example, patients 2 and 4 had decreased amikacin lung deposition (table 3), corresponding to worsening pulmonary function for patient 4 , but contrasting with improvement in pulmonary function for patient 2 (table 1). Both an increase in pharyngeal deposition and bronchial suppuration could have contributed to this apparently conflicting result.

Isotopic assessment of aerosol deposition is possible in experimental, but not in clinical conditions. Blood concentrations of aminoglycosides administered by nebulization are low or undetectable [4, 21], and several measurements would be necessary to assess the kinetics. This cannot be performed in clinical practice, especially in children. This study has demonstrated in 14 deposition, that the mass of amikacin excreted in the urine correlates with the mass of drug deposited in the lungs during nebulization. The average amount excreted in the urine represented $<16.0 \%$ of the mass deposited in the lungs (Uamik,total= (0.16 MDLmg)-6.21), indicating that a large amount of antibiotic is eliminated by mucociliary and digestive clearance and the gut. It can be speculated that the drug eliminated by the urine corresponded to the fraction of the drug deposited in the deep lungs, i.e. $16.0 \%$ of the MDLmg, that could be absorbed through the alveolar barrier. However, the distribution between plasma resorption and mucociliary clearance (i.e. 16.0 and $84.0 \%$, respectively) was only validated for $\mathrm{CF}$ patients chronically colonized by $P$. aeruginosa. For less severely ill $\mathrm{CF}$ patients, it can be speculated that the DF would be lower, and conversely that more peripheral deposition of the drug would lead to higher resorption. In a study performed to determine the pharmacokinetics of nebulized tobramycin in six cystic fibrosis patients, Touw et al. [22] showed that an average of $14.9 \%$ of the inhaled dose was excreted in the urine. However, they did not evaluate the mass deposited. This urinary pharmacokinetics approach remains to be validated in disease conditions other than cystic fibrosis.

As the reference clinical trial was performed with tobramycin [12], the question of the applicability of the present method for drugs other than amikacin remains crucial. Considering their pharmacological properties [23], tobramycin and amikacin are relatively small molecules, with very similar molecular weights (467 and 586 Daltons, respectively). Both are highly soluble in water. Their distribution volumes are also very close $\left(0.28-0.32 \mathrm{~L} \cdot \mathrm{kg}^{-1}\right.$ and $0.24-0.35 \mathrm{~L} \cdot \mathrm{kg}^{-1}$, respectively). All aminoglycosides are eliminated almost entirely by glomerular filtration. When administered parenterally, the fraction of the dose excreted during the first $24 \mathrm{~h}$ is $75-90 \%$ for tobramycin and $70-90 \%$ for amikacin, with renal clearances at $80-90$ and $80-120 \mathrm{~mL} \cdot \mathrm{min}^{-1}$, respectively. It therefore appears that pulmonary absorption, tissue distribution and renal excretion of both drugs are very similar. These findings suggest that the present results can reasonably be extrapolated to tobramycin.

The Bland \& Altman [10] analysis (fig. 3) showed a good statistical agreement between MDLmg and MDLmg,calc. However, due to the wide range of MDL, the standard deviation was as high as $29.7 \mathrm{mg}$.
The accuracy of MDLmg,calc could therefore be considered insufficient from a clinical point of view and for the follow-up of an individual patient.

However, the present report is the first to demonstrate a strong correlation between the mass of an aminoglycoside aerosol really deposited in the lungs, as measured by a validated but invasive and expensive method, and the excretion of this aerosol in the urine of cystic fibrosis patients. This suggests clinical implications for this urinary pharmacokinetics approach to monitor the dose-response relationship in groups of patients. Further studies are needed to establish its relevance for individual patient follow-up.

Acknowledgements. The authors are grateful to the patients and their parents for participating in this study, to K. Dieckman, C. Girault, C. Maurage, J.C. Rolland and F. Varaigne for recruiting patients, and to D. Raine for reviewing the English.

\section{References}

1. Touw DJ, Brimicombe RW, Hodson ME, Heijerman HGM, Bakker W. Inhalation of antibiotics in cystic fibrosis. Eur Respir J 1995; 8: 1594-1604.

2. Mukhopadhyay S, Singh M, Cater JI, Ogston S, Franklin M, Olver RE. Nebulised antipseudomonal antibiotic therapy in cystic fibrosis: a meta-analysis of benefits and risks. Thorax 1996; 51: 364-368.

3. Ramsey BW, Pepe MS, Quan JM, et al. for the Cystic Fibrosis Inhaled Tobramycin Study Group. Intermittent administration of inhaled tobramycin in patients with cystic fibrosis. $N$ Engl J Med 1999; 340: 23-30.

4. Ilowite JS, Gorvoy JD, Smaldone GC. Quantitative deposition of aerosolized gentamicin in cystic fibrosis. Am Rev Respir Dis 1987; 136: 1445-1449.

5. Shwachman H, Kulczycki L. Longterm study of one hundred and five patients with cystic fibrosis. Am J Dis Child 1958; 96: 6-15.

6. Quanjer PH, Tammeling GJ, Cotes JE, Pedersen OF, Peslin R, Yernault JC. Lung volume and forced ventilatory flows. Eur Respir J 1993; 6: S15-S40.

7. Dequin PF, Delatour F, Faurisson F, et al. Characterization on the bench of an amikacin aerosol before clinical studies. J Aerosol Med 1997; 10: 221230.

8. Bennett WD. Aerosolized drug delivery: fractional deposition of inhaled particles. J Aerosol Med 1991; 4: 223-237.

9. Smaldone GC, Fuhrer J, Steigbigel RT, McPeck M. Factors determining pulmonary deposition of aerosolized pentamidine in patients with human immunodeficiency virus infection. Am Rev Respir Dis 1991; 143: 727-737.

10. Bland JM, Altman DG. Statistical methods for assessing agreement between two methods of clinical measurement. Lancet 1986; i: 307-310.

11. Ansorg R, Müller KD, Wiora J. Comparison of inhibitory and bactericidal activity of antipseudomonal antibiotics againts Pseudomonas aeruginosa isolates from cystic fibrosis patients. Chemotherapy 1990; 36: 222-229.

12. Fiel SB. Aerosol delivery of antibiotics to the lower 
airways of patients with cystic fibrosis. Chest 1995; 107: 61S-64S

13. Shawar RM, MacLeod DL, Garber RL, et al. Activities of tobramycin and six other antibiotics against Pseudomonas aeruginosa isolates from patients with cystic fibrosis. Antimicrob Agents Chemother 1999; 43: 2877-2880.

14. Dally M, Kurrle S, Breslin A. Ventilatory effects of aerosol gentamicin. Thorax 1978; 33: 54-56.

15. Mellis CM, Levison H. Bronchial reactivity in cystic fibrosis. Pediatrics 1978; 61: 446-450.

16. Wonne R, Hofmann D, Posselt HG. Bronchial allergy in cystic fibrosis. Clin Allergy 1985; 15: 455-463.

17. Anderson PJ, Wilson JD, Hiller FC. Respiratory tract deposition of ultrafine particles in subjects with obstructive or restrictive lung disease. Chest 1990; 97: 1115-1120.

18. Sweeney TD, Skornik WA, Brain JD, Hatch V, Godleski JJ. Chronic bronchitis alters the pattern of aerosol deposition in the lung. Am J Respir Crit Care Med 1995; 151: 482-488.
19. Kim CS, Eldridge MA. Aerosol deposition in the airway model with excessive mucus secretions. $J$ Appl Physiol 1985; 59: 1766-1772.

20. Diot P, Palmer LB, Smaldone A, DeCelie-Germana J, Grimson R, Smaldone GC. RhDNase I aerosol deposition and related factors in cystic fibrosis. $\mathrm{Am}$ J Respir Crit Care Med 1997; 156: 1662-1668.

21. Palmer LB, Smaldone GC, Simon SR, O'Riordan TG, Cuccia A. Aerosolized antibiotics in mechanically ventilated patients: delivery end response. Crit Care Med 1998; 26: 31-39.

22. Touw DJ, Jacobs FAH, Brimicombe RW, Heuerman HGM, Bakker W, Breimer DD. Pharmacokinetics of aerosolized tobramycin in adult patients with cystic fibrosis. Antimicrob Agents Chemother 1997; 41: 184 187.

23. Humbert G, Leroy A, Oksenhendler G, Fillastre JP Aminoglycosides. In: Giroud JP, Mathé G, Meyniel $\mathrm{G}$, ed. Pharmacologie clinique, bases de la thérapeutique. 2nd ed. Paris, Expansion Scientifique Française, 1988; pp. 1399-1420. 